\title{
Effect of sitting position on respiratory status in preterm infants
}

\author{
Mika Shiraishi1 ${ }^{1,2, *}$, Kyoko Hirasawa ${ }^{1}$, Satoru \\ Shimizu $^{3}$, Hiroshi Nishida ${ }^{4}$ and Makiko Osawa ${ }^{1}$ \\ ${ }^{1}$ Department of Pediatrics, Tokyo Women's Medical \\ University, Japan \\ 2 Department of Infants' Brain and Cognitive \\ Development, Tokyo Women's Medical University, \\ Japan \\ ${ }^{3}$ Department of Hygiene and Public Health II, Tokyo \\ Women's Medical University, Japan \\ ${ }^{4}$ Maternal and Neonatal Center, Tokyo Women's \\ Medical University, Japan
}

\begin{abstract}
Objective: To evaluate whether using a sitting-type car safety seats for preterm infants is advisable.

Patients and methods: A total of 65 preterm infants underwent polysomnography in the supine and sitting positions. The infants with $<95 \%$ of $\mathrm{SpO}_{2}$ were assigned to the desaturation (DS) group. Their backgrounds, breathing patterns, and breathing types were analyzed. Results: Of the 65 cases, 18 were assigned to the DS group. No significant differences were observed between the DS and non-DS groups in their background characteristics. Of the 18 DS cases, 15 were included in the non-obstructive group (8, periodic breathing; 6 , tachypnea; 1, irregular breathing).

Conclusions: Immaturity of the respiratory center and decompensation for the respiratory changes caused by the sitting position were suspected to cause DS in infants. Sitting-type car safety seats should be used with caution for preterm infants, and all preterm infants need to be screened by polysomnographic examination in the sitting position.
\end{abstract}

Keywords: Car safety seat (CS); desaturation (DS); preterm infants; polysomnography; sitting position.

\footnotetext{
${ }^{*}$ Corresponding author:

Mika Shiraishi, MD

Pediatrics, Department of Infants' Brain and Cognitive Development

Tokyo Women's Medical University

8-1 Kawada-cho

Shinjuku-ku

Tokyo 162-8666

Japan

Tel.: + 81-3-3353-8111

Fax: +81-3-5269-7338

E-mail: mikas@ped.twmu.ac.jp
}

\section{Introduction}

In many countries, the use of a car safety seat (CS) is obligatory to ensure safety of infants and toddlers. A similar rule has been enforced in Japan since 2000. This rule applies to all children, including newborn babies and preterm infants while they are in a car.

Several studies have reported that the sitting position causes a decline in oxygen saturation $\left(\mathrm{SpO}_{2}\right)$ in preterm infants [21]. Willett et al. reported that, when infants were occasionally placed in the sitting position, the ratio of preterm infants with decreased $\mathrm{SpO}_{2}$ to that of term infants with decreased $\mathrm{SpO}_{2}$ was significantly higher [22]. Hypoxia may have important consequences, such as long-term neurological impairments and cognition disorders. Decreased IQ and delayed motor development have been reported in infants in whom the mean $\mathrm{SpO}_{2}$ declined to $<85 \%$, and attention-deficit hyperactivity disorder (ADHD) and impaired attention have occurred when the nadir $\mathrm{SpO}_{2}$ declined to $85-94 \%[6,11]$.

While it is clearly important to assess in detail the effect of the sitting position on respiration in neonates and preterm infants, there is no standard approach to doing so. In the United States, all preterm infants are recommended to undergo a monitoring examination when placed in a sitting-type CS, and the use of the flat, bedtype CS is advised for infants in whom hypoxia is detected [2-4]. However, no such unified recommendations or criteria exist in Japan. Moreover, in the United States, it is still controversial whether a monitoring examination is required for near-term infants [12], and no common decision has been reached regarding its subjects or methods.

In this study, preterm infants were examined by polysomnography, with the goal of determining the characteristics of those likely to be at a high-risk for hypoxia while in a sitting position. We also investigated whether high-risk infants could be identified by clinical charateristics or backgrounds. The overall goal of the study was to evaluate whether the use of a sitting-type CS for preterm infants is advisable and to develop a method for identifying those infants who, when placed in a sitting position, are at high-risk for a decreased $\mathrm{SpO}_{2}$.

\section{Methods}

\section{Subjects}

Preterm infants were recruited from the Maternal and Neonatal Center, Tokyo Women's Medical University, Tokyo, Japan, 
between August 2005 and November 2006. A total of 65 preterm infants underwent polysomnography in the sitting position when they were eligible for hospital discharge. None of the infants exhibited any abnormal findings in the preliminary polysomnography in the supine position. The institutional review board approved the present study, and written informed consent was obtained from the parents before the examination. The characteristics of the 65 preterm infants ( 36 boys and 29 girls) were as follows: gestational ages ranged from 24 to 36 weeks ( $30.3 \pm 3.8$ weeks, median $=32.3$ weeks), birth weights ranged from 558 to $3128 \mathrm{~g}(1502.7 \pm 651.9 \mathrm{~g}$, median=1440.0 g), and post-conceptional age at examination ranged from 36 to 46 weeks $(40.5 \pm 2.5$ weeks, median $=39.9$ weeks). Exclusion criteria consisted of the presence of any congenital or chromosomal abnormality, prenatal brain lesions (e.g., cysts and infarctions), any clinical symptoms indicating neurological disorders at the time of examination, and chronic lung disease requiring oxygen therapy.

\section{Study protocol}

The 65 selected preterm infants were maintained in the supine position in a bed-type CS after feeding and underwent polysomnography. Subsequently, these infants, who had no abnormal findings during this examination, underwent a second round of polysomnography, this time while positioned in a sitting-type CS.

The CS was manufactured by Graco (SnugRide; Graco, Minneapolis, MN, USA); it was positioned at $45^{\circ}$ relative to the horizontal, and the interior angle of the sitting-type CS was $120^{\circ}$. As the aim of this study was to investigate the effect of the sitting posture alone on polysomnographic respiratory changes, each infant was gently placed in the center of the seat to exclude any factors that might affect the natural sitting position, such as a roll of towels or harness clips. Any other type of equipment did not support the infant's head or torso.

Each infant was studied for at least 30 min until one or more sleep cycles appeared. The study was discontinued when serious changes in vital signs, such as decreases in $\mathrm{SpO}_{2}$ ( $<70 \%$ at any time, or $<90 \%$ persistent for $5 \mathrm{~min}$ ), bradycardia ( $<90 \mathrm{bpm}$ ), severe tachypnea ( $>60 / \mathrm{min})$, and/or retraction were observed; however, these discontinued cases were included in the analysis.

\section{Infant monitoring}

Polysomnographic recordings (Sandman; Tyco Healthcare, Mansfield, MA, USA) were obtained using terminals attached to record the heart rates. An oronasal thermistor recorded airflow. A barosensitive pickup band recorded abdominal wall movement, and a pulse oximeter ( $\mathrm{N}-180$, Nellcor Puritan Bennett, Hayward, CA; averaging time was $12 \mathrm{~s}$ ) attached to a toe measured the $\mathrm{SpO}_{2}$. A scalp encephalogram (EEG) was obtained using unipolar leads $\mathrm{C} 3, \mathrm{C} 4, \mathrm{O} 1$, and $\mathrm{O} 2$; the ear-lobule leads $\mathrm{A} 1$ and $\mathrm{A} 2$ were used as indifferent electrodes. In addition, an electrooculogram (EOG) and a chin electromyogram (EMG) were recorded.

\section{Analysis}

Infants with $\mathrm{SpO}_{2}<95 \%$ were assigned to the desaturation (DS) group; the remaining infants were assigned to the non-DS group.
DS was defined as follows: the start point was set at the beginning of an $\mathrm{SpO}_{2}$ decline that continued for $>5 \mathrm{~s}$, and the end point was defined as the point when the $\mathrm{SpO}_{2}$ recovered and attained a value $>95 \%$.

However, there were cases with a decline in $\mathrm{SpO}_{2}$ after recovery. Declines in $\mathrm{SpO}_{2}$ occurring within $5 \mathrm{~s}$ and accompanied by an abnormal breathing pattern were considered to be cases of DS and regarded as a continuation of the previous episode. The DS patterns were classified into two groups based on the observed respiratory disorder pattern. Obstructive DS was defined as the recording of abdominal movement without nasal airflow, and non-obstructive DS as the condition under which nasal airflow was synchronized with abdominal movement. Infants with non-obstructive DS were further sorted into three groups based on the precedent breathing pattern: (1) tachypnea (respiratory rates $>60 / \mathrm{min}$ ), (2) periodic breathing (pause in breathing $>3 \mathrm{~s}$, occurring three or more times in $20 \mathrm{~s}$ ), and (3) irregular breathing (irregular breathing movements with pauses). Infants with non-obstructive DS were sorted into three groups according to the pattern of DS: episodic, persistent, and gradient types. (1) The episodic type was defined as DS with a recovery that took place within $30 \mathrm{~s}$. (2) The persistent type was defined as DS with a recovery that took $>30 \mathrm{~s}$. These two types were independent and non-overlapping. Within the persistent type, if the $\mathrm{SpO}_{2}$ decline persisted for more than $3 \mathrm{~min}$, the condition was defined as prolonged-type non-obstructive DS. (3) Gradient-type non-obstructive DS reflected a gradual decline of the $\mathrm{SpO}_{2}$ baseline. It was defined as the average $\mathrm{SpO}_{2}$ value from $5 \mathrm{~min}$ before the end of the measurement to termination, with a difference $\geq 5 \%$ from the average $\mathrm{SpO}_{2}$ value from the start of measurement to 5 min afterwards.

The sleep phase was identified by visual analyses of four distinct EEG patterns [5, 8], namely, mixed frequency (active sleep), low-voltage irregular (active sleep), trace alternant (quiet sleep), and high-voltage slow (quiet sleep). Transitional sleep occurred within and between these sleep segments.

\section{Statistical analysis}

Background characteristics were compared between the DS and non-DS groups: birth weight, gestational age in weeks (GA) at birth, APGAR score, and post-conceptional age in weeks (PCA) at examination.

The infants' clinical courses were also compared. Respiratory distress syndrome (RDS; requiring surfactant administration), chronic lung disease (CLD; requiring oxygen therapy for more than 28 days), and apnea were considered as breathing disorders. Patent ductus arteriosus (PDA; requiring indomethacin administration) and retinopathy of prematurity ( $R O P$; requiring laser therapy) are complications likely to occur in premature infants.

A $\chi^{2}$ analysis (with Yates' continuity correction, when required) was used for calculating the significance of the differences among the groups. Student's $t$-test or Welch's $t$-test was used for individual comparisons. The normality of the distribution was determined, as was the appropriateness of Student's $t$-test or Welch $t$-test for the statistical population. $\mathrm{P}<0.05$ was considered statistically significant.

\section{Akaike's information criterion}

Akaike's information criterion (AIC), an unbiased estimate of the mean expected log likelihood, is a reasonable criterion for 
comparing statistical models under mild conditions. The mean expected log likelihood is a reasonable criterion for comparing statistical models. A smaller AIC implies a better fit of the model to the given data through an autoregressive process. Minimum $\mathrm{AIC}$ is used to determine the value.

\section{Results}

Of the 65 infants studied, 47 were included in the nonDS group and 18 in the DS group. A correlation was observed between GA and PCA in both DS and non-DS groups (Figure 1).

The DS and non-DS groups did not differ significantly with respect to background characteristics: GA at birth, birth weight, PCA at examination, and number of days elapsed after birth at the time of examination. No significant difference was recognized between the two groups in the clinical course of these infants (Table 1). An analysis based on the infants' GA is shown in Figure 2. Since GA ranged between 24 and 36 weeks, we divided the values into those obtained in the first half (i.e., weeks

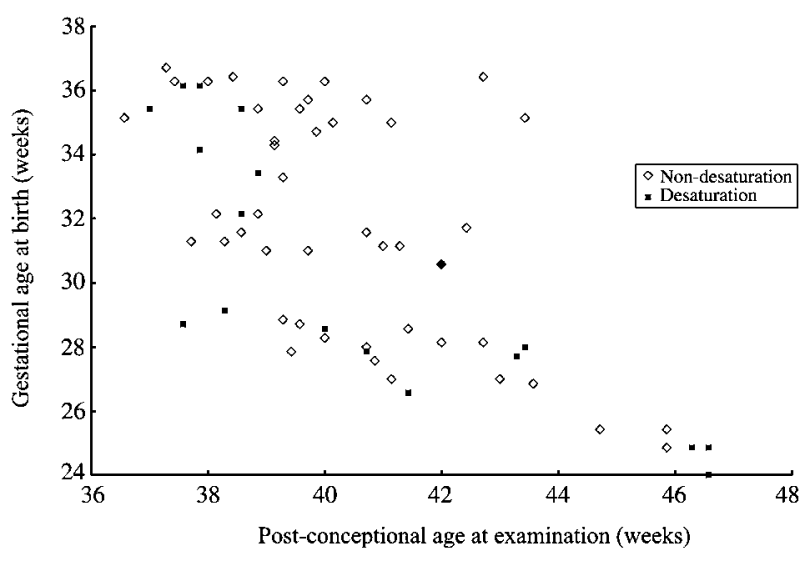

Figure 1 The DS ratio tends to be higher at an earlier GA or PCA.

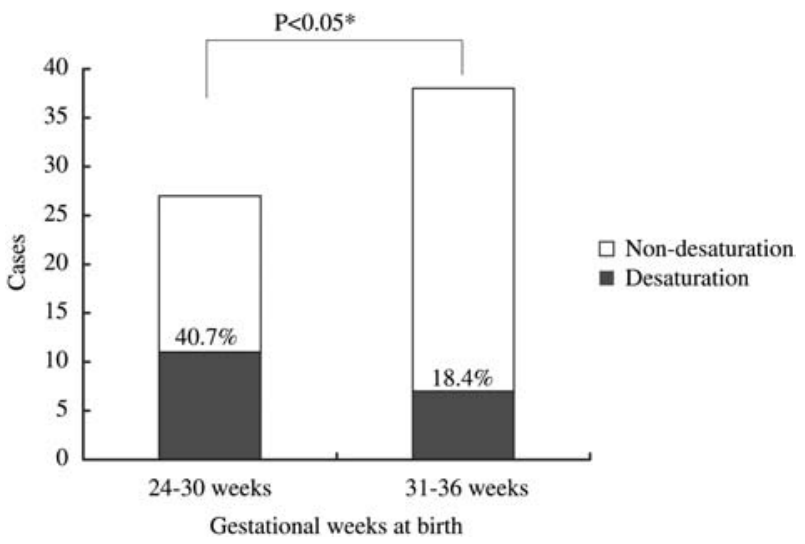

Figure 2 Infants in the study were divided into two groups according to their GA: those with a GA of 24-30 weeks and those with a GA of 31-36 weeks. The occurrence of DS was determined in each group and the results of the two groups were compared.

24-30), and those obtained in the last half (i.e., weeks 31-36), and then compared them. The ratio of infants in whom DS was observed to those who did not experience DS was significantly higher in the group born before 31 gestational weeks than in the group born after 31 gestational weeks (Figure 2).

Figure 3 presents an analysis of the effect of PCA. As the minimal AIC was at week 38.7, PCA was divided into $<38$ weeks and $\geq 38$ weeks. The ratio of infants with DS to those without DS in the group examined at $<38$ weeks of PCA was significantly higher than in the group examined at $\geq 38$ weeks of PCA.

Of the 18 infants with DS, 15 (83.3\%) belonged to the non-obstructive group. In-depth data for the nonobstructive DS group are shown in Table 2. Analysis of the precedent breathing pattern showed that eight of the 15 infants had periodic breathing, six infants had tachypnea, and one infant had irregular breathing. Tachypnea occurred in five of the six (83.3\%) preterm infants with a

Table 1 Comparison of the background factors between the non-desaturation group and the desaturation group.

\begin{tabular}{llll}
\hline & $\begin{array}{l}\text { Non-desaturation } \\
(\mathrm{n}=47)\end{array}$ & $\begin{array}{l}\text { Desaturation } \\
(\mathrm{n}=18)\end{array}$ & P-value \\
\hline Sex (male:female) & $25: 22$ & $11: 7$ & $>0.2$ \\
Post conceptional age (PCA) at examination & $40.4 \pm 2.1$ weeks & $40.7 \pm 3.3$ weeks & $>0.2$ \\
Days after birth at examination & $59.9 \pm 36.7$ days & $75.9 \pm 48.6$ days & $>0.2$ \\
Gestational weeks (GA) at birth & $31.8 \pm 3.6$ weeks & $30.2 \pm 4.1$ weeks & $>0.2$ \\
Birth weight & $1559.0 \pm 637.6 \mathrm{~g}$ & $1355.6 \pm 684.3 \mathrm{~g}$ & $>0.2$ \\
APGAR score (1 min) & $7.0 \pm 1.8$ & $5.8 \pm 2.9$ & 0.120 \\
APGAR score (5 min) & $8.5 \pm 1.4$ & $7.4 \pm 2.3$ & 0.093 \\
Postnatal & & & $>0.2$ \\
RDS & $9(19.1 \%)$ & $7(38.9 \%)$ & $>0.2$ \\
CLD & $18(38.3 \%)$ & $10(55.6 \%)$ & $>0.2$ \\
Apnea & $23(48.9 \%)$ & $11(61.1 \%)$ & $>0.2$ \\
PDA & $7(14.9 \%)$ & $4(22.2 \%)$ & $>0.2$ \\
ROP & $6(12.8 \%)$ & $4(22.2 \%)$ & \\
\hline
\end{tabular}




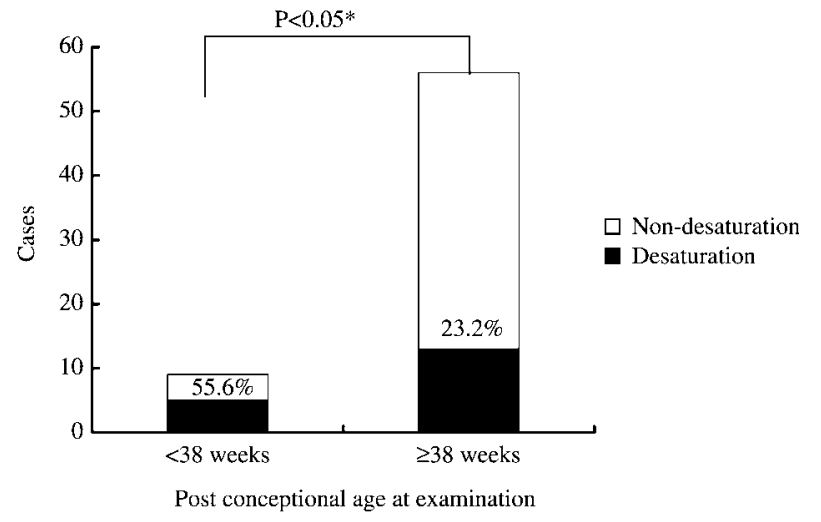

Figure 3 In infants with a PCA of $<38$ weeks, the incidence of DS was $>50 \%$.

GA $<28$ weeks. The most frequently observed DS pattern was the episodic type. Moreover, infants in whom episodic-type DS occurred frequently often had complications of persistent-type DS. Four of the eight infants (50\%) with persistent-type DS had complications of gradient-type DS. Complications of gradient-type DS occurred in four of the six infants $(66.7 \%)$ born before they reached 28 weeks and three of the six infants $(50 \%)$ with tachypnea. Furthermore, four of the eight infants (50\%) with persistent-type DS had complications of the prolonged type.

With respect to the relationship between the precedent breathing pattern and the sleep phase, DS occurred during the active/transitional sleep stage in five of the eight infants with periodic breathing (62.5\%). Consequently, in periodic breathing, the breathing pattern was assumed to be associated with the sleep phase. In infants with tachypnea, however, DS was observed equivalently in the active/transitional and quiet sleep stages. In infants with tachypnea, the breathing pattern had a less significant relationship with the sleep phase.

\section{Discussion}

None of the infants in this study showed abnormal findings, such as reduced $\mathrm{SpO}_{2}$, in the preliminary polysomnography conducted in the supine position (interior angle: $178^{\circ}$ ) [21, 22]. However, approximately $30 \%$ of the preterm infants in our study experienced DS in the sitting position and were thus considered to be at high risk for hypoxia. According to previous reports, ADHD and impaired attention are associated with a decline in the nadir $\mathrm{SpO}_{2}$ to between 85 and $94 \%[6,11]$. We therefore consider that the $\mathrm{SpO}_{2}$ of infants should be maintained at $>95 \%$.

One of the main purposes of this study was the development of a rapid and efficient screening method to identify infants at high risk of DS in order to avoid a dangerous decline of $\mathrm{SpO}_{2}$ to $<85 \%$. During the relatively short examination time of approximately $30 \mathrm{~min}$ used in this study, we observed prolonged ( $>3 \mathrm{~min}$ ) and/or frequent DS $\left(<90 \% \mathrm{SpO}_{2}\right)$ in approximately $40 \%$ of the patients (7 of 18 cases). The DS group included infants who required careful clinical attention during examination, such as one subject whose $\mathrm{SpO}_{2}$ declined to nearly $70 \%$ and remained low (GA, 24 weeks +4 days; male) and another whose baseline $\mathrm{SpO}_{2}$ declined immediately after being placed in the sitting position and remained low (GA, 36 weeks +6 days; male). In the latter infant, the examination was discontinued at 12 min because his $\mathrm{SpO}_{2}$ remained low without recovery. The background characteristics were not significantly different between the DS and non-DS groups. Presumably, if the examination time were extended, more cases of DS

Table 2 In-depth data for the non-obstructive DS group $(n=15)$.

\begin{tabular}{|c|c|c|c|c|c|c|}
\hline \multirow[t]{2}{*}{ No. } & \multirow[t]{2}{*}{ GA (W) } & \multirow[t]{2}{*}{ DS pattern } & \multirow[t]{2}{*}{ Sleep stage } & \multicolumn{2}{|c|}{ DS type } & \multirow[t]{2}{*}{ Gradient } \\
\hline & & & & Episodic (times) & Persistent (times) & \\
\hline 1 & 24.0 & Tachypnea & Quiet & 8 & - & - \\
\hline 2 & 24.9 & Tachypnea & Quiet & 12 & $2^{*}$ & + \\
\hline 3 & 24.9 & Tachypnea & Active & 17 & $8^{*}$ & + \\
\hline 4 & 26.6 & Tachypnea & Quiet & 19 & $2^{*}$ & + \\
\hline 5 & 27.7 & Tachypnea & Transitional & 5 & - & - \\
\hline 6 & 27.9 & Periodic & Active & 10 & - & + \\
\hline 7 & 28.0 & Periodic & Active & 5 & - & - \\
\hline 8 & 29.1 & Periodic & Active & 34 & 5 & - \\
\hline 9 & 30.6 & Tachypnea & Active & 25 & 8 & - \\
\hline 10 & 32.1 & Periodic & Quiet & 6 & - & - \\
\hline 11 & 34.1 & Periodic & Quiet & 38 & 3 & - \\
\hline 12 & 35.4 & Periodic & Active & 27 & 1 & - \\
\hline 13 & 35.4 & Periodic & Transitional & 3 & - & - \\
\hline 14 & 36.1 & Periodic & Quiet & 9 & 6 & $+<$ Discontinued at $12 \min >$ \\
\hline 15 & 36.1 & Irregular & Quiet & 0 & All course ${ }^{*}$ & $+<$ Discontinued at $16 \min >$ \\
\hline
\end{tabular}

*Prolonged DS. 
would be detected. Therefore, the definition of DS as $<95 \% \mathrm{SpO}_{2}$ represents a useful cut-off value for quickly (in approximately $30 \mathrm{~min}$ ) identifying infants with a high risk of developing DS.

In this study, infants at a high risk for DS when placed in the sitting position were difficult to identify by clinical history alone. Therefore, all preterm infants should undergo a polysomnography in order to determine the safety of a sitting-type CS.

Several previous reports concerning the occurrence of DS in infants placed in the sitting position emphasized this position as the cause of obstruction [20]. However, in the present study on preterm infants, most cases of DS were of the non-obstructive type. Of the 15 infants with DS, eight had periodic breathing, always of the episodic-type. Four of the eight infants were recognized as having persistent-type complications whereas prolonged-type DS was not observed. An analysis of the sleep phase during which DS occurred showed that five of these eight infants were in the active/transitional sleep stage at the time of DS.

Generally, periodic breathing is the characteristicbreathing pattern in preterm infants, and it often occurs in the active/transitional sleep stage [15, 17]. In preterm infants, DS with periodic breathing sometimes occurs before a PCA of 36 weeks. Since the general condition of the study infants was stable at a PCA of approximately 40 weeks, DS was not observed in this group, although periodic breathing persisted. The majority of the preterm infants included in this study had a PCA of 37 weeks at examination, a stable general condition, and no DS episodes in the supine position. DS occurred in the sitting position following periodic breathing during the active/ transitional sleep stage, which is considered to be the characteristic respiratory pattern in preterm infants. Elder et al. reported that neonatal respiratory instability following discharge from the hospital was more likely to be associated with immaturity than with lung conditions such as CLD [9]. The preterm infants in the present study did not appear to have any respiratory problems, such as DS episodes in the spine position. However, we speculate that the premature respiratory center had difficulty maintaining adequate ventilation by means of periodic breathing when these infants were placed in the sitting position, that is, against gravity.

In infants with non-obstructive DS, the other major breathing pattern was tachypnea. Of the 15 infants with non-obstructive DS, tachypnea was recorded in 6 $(40.0 \%), 5(83.3 \%)$ of whom were born before a GA of 28 weeks. As DS occurred equivalently in active/transitional and quiet sleep (three infants each), tachypnea seems to correlate poorly with sleep stage. In the early stages of development, an infant's chest wall has a high compliance. Therefore, the chest wall of infants are easily deformed, and the alveoli are easily compressed because they cannot counter the reactionary constriction after lung expansion. As a result, functional end-tidal lung volume in infants is frequently decreased [10]. If these infants are placed in the sitting position, the chest-wall is deformed and ventilation restricted due to the abdominal compression caused by flexion of the hip joint, which forces the diaphragm upward. Tachypnea ensues as a compensatory mechanism to maintain ventilation in response to the decreased tidal volume. Of the six infants with tachypnea, four (66.7\%) had persistent-type DS, including three $(75.0 \%)$ with complications of prolongedtype DS. Three of the tachypneic infants $(50 \%)$ had gradient-type DS, in each case complicated by prolonged-type DS. Because most of the infants with tachypnea were born at $<28$ weeks, and several infants with gradient-type DS had complications of prolonged-type DS, easy fatigability caused by poor bodily condition seems to be an important factor contributing to the development of DS.

Hypoxia in children is reported to have not only shortterm cardiovascular effects, such as bradycardia and apnea [16], but also long-term effects on neurological development and cognitive function. Long-term effects dependent on $\mathrm{SpO}_{2}$ levels have been demonstrated in previous studies [6]. At $\mathrm{SpO}_{2}$ levels $<85 \%$, developmental disorders such as delayed motor development (mean $\mathrm{SpO}_{2} 77.3 \%$ [1]) and decreased IQ (mean $\mathrm{SpO}_{2}$ 82 [18] to $84 \%$ [23]) occurred more frequently than impaired attention (mean $\mathrm{SpO}_{2} 66.1 \%$ [14]). At $\mathrm{SpO}_{2}$ levels between 85 and $94 \%$, behavioral disorders such as impaired attention, along with lower IQ (nadir $\mathrm{SpO}_{2}$ 90.7\% [7]) and ADHD (nadir $\mathrm{SpO}_{2} 90.9 \%$ [13] to $95 \%$ [19]) have been reported. Janvier et al. suggested that the number of days on which apnea was recorded during hospitalization was directly associated with the severity of outcome: the greater the number of days, the worse the outcome. Among the potential explanations for this finding is that multiple recurrent hypoxic and bradycardic spells may cause brain injury [11]. For this reason, persistent-type DS, particularly prolonged-type DS has important clinical implications.

All the infants with DS had the episodic type, except one infant, who persistently had irregular breathing. Moreover, persistent-type DS frequently complicated the episodic type. The four infants with prolonged-type DS had complications of gradient-type DS; three of these four were born before 28 weeks.

We observed that the earlier the GA at birth or PCA at examination, the higher the proportion of DS-affected infants. Furthermore, the proportion of infants with DS was significantly higher within the group born at $<31$ weeks. Therefore, such infants should be carefully examined as should preterm infants with a PCA of $<38$ weeks at examination.

Many near-term infants (GA at birth 34-36 weeks) were included in the group of infants with a PCA of $<38$ weeks at examination. The general condition of these near-term 
infants stabilized relatively quickly, and they often could be examined by polysomnography during their early days of life. Therefore, the proportion of DS in near-term infants was higher because they tended to undergo examination at an early PCA. Based on the above findings, polysomnography in the sitting position should be performed at a PCA of $>38$ weeks, i.e., when the general condition of preterm infants has stabilized.

\section{References}

[1] Aisenberg RB, Rosenthal A, Nadas AS, Wolff PH. Developmental delay in infants with congenital heart disease. Correlation with hypoxemia and congestive heart failure. Pediatr Cardiol. 1982;3:133-7.

[2] American Academy of Pediatrics, Committee on Injury and Poison Prevention on Fetus and Newborn. Safe transportation of premature and low birth weight infants. Pediatrics. 1996;97:758-60.

[3] American Academy of Pediatrics, Committee on Injury and Poison Prevention. Safe transportation of newborn at hospital discharge. Pediatrics. 1999;104:986-7.

[4] American Academy of Pediatrics, Committee on Injury and Poison Prevention on Fetus and Newborn. Selecting and using the most appropriate car safety seats for growing children: guidelines for counseling parents. Pediatrics. 2002;109:550-3.

[5] Anders T, Emde R, Parmelee A. A manual of standardized terminology, technique, and criteria for scoring of state of sleep and wakefulness in newborn infants. Los Angeles, CA: UCLA BIS/BRI publications office NINDS Neurological Information Network; 1971.

[6] Bass JL, Corwin M, Gozal D, Moore C, Nishida H, Kinane TB. The effect of chronic or intermittent hypoxia on cognition in childhood: a review of the evidence. Pediatrics. 2004;114:805-16.

[7] Blunden S, Lushington K, Kennedy D, Martin J, Dawson D. Behavior and neurocognitive performance in children aged 5-10 years who snore compared to controls. J Clin Exp Neuropsychol. 2000;22:554-68.

[8] Carrozzi M, Accardo A, Bouquet F. Analysis of sleep-stage characteristics in full-term newborns by means of spectral fractal paraments. Sleep. 2004;27:1384-93.

[9] Elder DE, Campbell AJ, Doherty DA. Prone or supine for infants with chronic lung disease at neonatal discharge? J Paediatr Child Health. 2005;41:180-5.

[10] Frappell PB, Macfarlane PM. Development of mechanics and pulmonary reflexes. Respir Physiol Neurobiol. 2005 149:143-54.
[11] Janvier A, Khairy M, Kokkotis A, Cormier C, Messmer D, Barrington KJ. Apnea is associated with neurodevelopmental impairment in very low birth weight infants. J Perinatol. 2004;24:763-8.

[12] Merchant JR, Worwa C, Porter S, Coleman JM, deRegnier RA. Respiratory instability of term and near-term healthy newborn infants in car safety seats. Pediatrics. 2001;108: 647-52.

[13] O'Brien LM, Holbrook CR, Mervis CB, Klaus CJ, Bruner JL, Gozal D. Sleep and neurobehavioral characteristics of 5- to 7-year-old children with parentally reported symptoms of attention-deficit/hyperactivity disorder. Pediatrics. 2003;111:554-63.

[14] O'Dougherty M, Nuecheterlein KH, Drew B. Hyperactive and hypoxic children: signal detection, sustained attention, and behavior. J Abnorm Psychol. 1984;93:178-91.

[15] Oliveira AJ, Nunes ML, Fojo-Olmos A, Reis FM, de Costa JC. Clinical correlates of periodic breathing in neonatal polysomnography. Clin Neurophysiol. 2004;115:2247-51.

[16] Poets CF, Stebbens VA, Samuels MP, Southall DP. The relationship between bradycardia, apnea, and hypoxia in preterm infants. Pediatr Res. 1993;34:144-7.

[17] Razi NM, DeLauter M, Pandit PB. Periodic breathing and oxygen saturation in preterm infants at discharge. J Perinatol. 2002;22:442-4.

[18] Silbert A, Wolff PH, Mayer B, Rosenthal A, Nadas AS. Cyanotic heart disease and psychological development. Pediatrics. 1969;43:192-200.

[19] Stradling JR, Thomas G, Warley AR, Williams P, Freeland A. Effect of adenotonsillectomy on nocturnal hypoxaemia, sleep disturbance, and symptoms in snoring children. Lancet. 1990;335:249-53.

[20] Tonkin SL, Mclntosh CG, Hadden W, Dakin C, Rowley S, Gunn AJ. Simple car seat insert to prevent upper airway narrowing in preterm infants: a pilot study. Pediatrics. 2003;112:907-13.

[21] Willett LD, Leuschen MP, Nelson LS, Nelson RM Jr. Risk of hypoventilation in premature infants in car seats. $J$ Pediatr. 1986;109:245-8.

[22] Willett LD, Leuschen MP, Nelson LS, Nelson RM Jr. Ventilatory changes in convalescent infants positioned in car seats. J Pediatr. 1989;115:451-5.

[23] Wright M, Nolan T. Impact of cyanotic heart disease on school performance. Arch Dis Child. 1994;71:64-70.

The authors stated that there are no conflicts of interest regarding the publication of this article.

Received July 6, 2008. Revised December 17, 2008. Accepted January 26, 2009. Previously published online March 23, 2009. 